\title{
Effects of Continuous Passaging on Mineralization of MC3T3-E1 Cells with Improved Osteogenic Culture Protocol
}

\author{
Xiang-Zhen Yan, MSc, ${ }^{1,2, *}$ Wanxun Yang, MSc, ${ }^{1,3, *}$ Fang Yang, PhD, ${ }^{1}$ Monique Kersten-Niessen, BSc, \\ John A. Jansen, PhD, DDS, and Sanne K. Both, PhD ${ }^{1}$
}

The murine-derived MC3T3-E1 cell line provided by the American Type Culture Collection (ATCC) is a wellknown osteogenic cell culture model system to test materials in vitro. However, the effect of passaging on its mineralization capacity has never been described and their culture supplements can be further optimized. Therefore, we evaluated the influence of the passage number and different osteogenic culture supplements, including ascorbic acid (AsAP) and dexamethasone (Dex) on the osteogenic capacity of MC3T3-E1 cells. This capacity was measured by the deposited calcium, the alkaline phosphatase activity, and the expression of osteogenic-related genes, including bone sialoprotein $(B S P)$, osteocalcin $(O C)$, and osteopontin $(O P N)$. The results indicated that the mineralization capacity of MC3T3-E1 cells significantly decreased during passaging and got exhausted at passage 34 , as assessed by measuring calcium deposition after 28 days of osteogenic induction. Moreover, the combination of AsAP and Dex triggered significantly more mineralization in MC3T3-E1 cells than the ATCC recommended addition of AsAP alone, as indicated by increased calcium deposition and higher expression of BSP and OPN. However, Dex alone could not trigger this effect, but only in combination with the AsAP, which indicates that Dex has no direct effect on mineralization. In conclusion, the passage number of MC3T3-E1 cells is of great importance and the use of cells above 30 passages should be avoided. In addition, the favored osteogenic supplements providing an improved osteogenic differentiation of MC3T3-E1 cells are the combination of AsAP and Dex.

\section{Introduction}

$\mathrm{C}$ ELL LINES ARE important tools for scientific research as the cells can conveniently be grown indefinitely in culture. The MC3T3-E1 cell line established from newborn mouse calvaria is a well-known in vitro osteogenic model system and has been widely used in bone tissue engineeringrelated research. ${ }^{1}$ In general, osteoblast development has been subdivided into three stages; proliferation, extracellular matrix maturation, and mineralization. ${ }^{2}$ MC3T3-E1 cells display a sequential development pattern of proliferation and differentiation, resulting in calcified bone tissue similar to in vivo bone formation. ${ }^{3}$ A series of subclones have been isolated from the MC3T3-E1 cell line and subclone 4, which shows high osteogenic potential are very useful to study in vitro osteogenesis. ${ }^{4}$

However, it is known that the number of passages can influence cellular function. Significantly decreased differen- tiation potentials of mesenchymal stem cells due to passaging have been observed. ${ }^{5}$ For example, a previous study showed that undifferentiated rat bone marrow cells lost their osteogenic potential after three times of passaging. ${ }^{6}$ In another study, it was demonstrated that the osteogenic capacity of adipose-derived stem cells also gradually decreases during long-term manipulation. ${ }^{7}$ This can also occur with cell lines, which involves the risk that they are used beyond safe passage. ${ }^{8}$ This is important because it is known that in studies concerning osteogenesis, and in which MC3T3-E1 cells were used, the passage number was not always clearly defined, which is an obstacle to make an objective evaluation and comparison among different studies. Cell lines are usually obtained from colleagues or another source without the information of the passage number, increasing the likelihood of leading to inaccurate or irreproducible results. ${ }^{8}$ The osteoblastic function of MC3T3-E1 cells, as measured by osteocalcin (OC) secretion and alkaline phosphatase (ALP)

\footnotetext{
${ }^{1}$ Department of Biomaterials, Radboud University Nijmegen Medical Center, Nijmegen, The Netherlands.

${ }^{2}$ Department of Stomatology, Shandong University, Jinan, P. R. China.

${ }^{3}$ Key Laboratory for Oral Biomedical Engineering of Ministry of Education, School and Hospital of Stomatology, Wuhan University, Wuhan, P. R. China.

*The two authors contributed equally to this work.
} 
activity, decreases significantly at late passage $(>65)$ as compared to the early passage $(<20) .^{9}$ Additionally, population doubling time of MC3T3-E1 cells declines from $15 \mathrm{~h}$ at passage 30 to $20 \mathrm{~h}$ at passage $65 .^{10}$ However, the mineralization ability of MC3T3-E1 cells during continuous passaging, as measured by the deposited amount of calcium has not been reported, and whether there exists a difference in the osteoblastic function between early and late passages still needs to be clarified. Another question of importance is how often MC3T3-E1 cells can be passaged before they lose their osteogenic capacity, so that the cells can be used for research without over-passaging.

In addition to the passage number, the culturing conditions are also essential for osteogenic differentiation. It is well known that the presence of beta-glycerophosphate $(\beta G P)$ is required for the mineralization of mature cultures. ${ }^{3}$ In addition, The most widely used osteogenic supplements include ascorbic acid (AsAP) and dexamethasone (Dex). AsAP is essential for the maturation and deposition of collagen and it contributes to the induction of ALP activity. ${ }^{3}$ It has been reported that MC3T3-E1 cells exhibit high levels of osteoblast differentiation with the addition of $\beta G P$ and AsAP. ${ }^{4}$ Therefore, the combination of these two supplements is recommended by the American Type Culture Collection (ATCC) as the osteogenic stimulator for MC3T3E1 cells. Another frequently used osteogenic inducer is Dex, which has been employed for osteogenic induction of a variety of cells, including mesenchymal stem cells, ${ }^{11,12}$ gingival fibroblasts, ${ }^{13}$ periosteal-derived cells, ${ }^{14}$ and dental follicle cells. ${ }^{15}$ Dex treatment can increase the ALP activity of mesenchymal stem cells, and was essential for their mineralization of the extracellular matrix. ${ }^{12}$ Till now, the effect of Dex on the osteogenic ability of MC3T3-E1 cells has not been described. In addition, the osteogenic capacity of different combinations of these culturing supplements remains to be investigated.

Based on the aforementioned research gaps, the aim of the present study was to define the effects of continuous passaging and composition of culturing supplements on the osteogenic ability of MC3T3-E1 cells.

\section{Materials and Methods}

\section{Cell culture and seeding}

MC3T3-E1 Subclone four cells (ATCC CRL-2593) from passage 16 were cultured in a proliferation medium containing the $\alpha$ MEM (Gibco) supplemented with 10\% FCS (Gibco) and 100 units/mL pen/strep (Gibco) in a humidified incubator set at $37^{\circ} \mathrm{C}$ and $5 \% \mathrm{CO}_{2}$. Upon $80 \%$ confluency, cells were detached using trypsin/EDTA $(0.25 \% \mathrm{w} / \mathrm{v}$ tryp$\sin / 0.02 \%$ EDTA) and continuously subcultured at a density of 3500 cells $/ \mathrm{cm}^{2}$ for 3 days or 2500 cells $/ \mathrm{cm}^{2}$ for 4 days. The cells were expanded for five passages and afterward frozen in the $\alpha$-MEM (Gibco) containing 20\% FCS (Gibco) and 10\% DMSO (Sigma) at passage 21 in liquid nitrogen for future use. From passage 22, cells were seeded every four passages (passage 22, 26, 30, 34, 38, 42, 46, and 50) at a density of 5000 cells/well in 24-well plates, and 10,000 cells/well in12-well plates in a control medium [proliferation medium $+10 \mathrm{mM}$ $\beta$ GP (Sigma)], control medium $+50 \mu \mathrm{g} / \mathrm{mL}$ AsAP (Sigma), control medium $+10 \mathrm{nM}$ Dex (Sigma), or control medi$\mathrm{um}+\mathrm{combination}$ of AsAP and Dex. On days 1, 4, and 7, the morphology of the cells was observed by an inverse phasecontrast microscope (Leica DMIL) at $\times 10$ magnification.

\section{Cell viability}

The viability of cells from passage 22 through passage 50 was evaluated every four passages using a Live/Dead Viability/Cytotoxicity Kit (Molecular Probes) after 2 days of culture. Live cells were labeled with calcein AM and dead cells were labeled with ethidium homodimer. Cells were washed in phosphate-buffered saline (PBS), incubated in $2 \mathrm{mM}$ calcein-AM and $4 \mathrm{mM}$ ethidium homodimer in PBS for $30 \mathrm{~min}$ at $37^{\circ} \mathrm{C}$, rinsed in PBS for two times, and then observed by using Zeiss Imager Z1 together with the AxioCam MRc5 camera using AxioVision 4.6.3 software (Carl Zeiss Microimaging $\mathrm{GmbH}$ ).

\section{Calcium content}

After 14, 21, and 28 days of culture, the amount of deposited calcium by MC3T3-E1 cells in 24-well plates was quantified $(n=4)$ using the ortho-cresolphthalein complexone method (Sigma). Briefly, cells were washed twice with PBS, and then incubated in $1 \mathrm{~mL}$ of $0.5 \mathrm{~N}$ acetic acid on a shaking platform overnight. For analyses, a $10 \mu \mathrm{L}$ sample or standard was incubated with $300 \mu \mathrm{L}$ of work reagent in a 96well plate at room temperature for $10 \mathrm{~min}$. The standards $(0-100 \mu \mathrm{g} / \mathrm{mL})$ were generated by serial dilutions of a $\mathrm{CaCl}_{2}$ stock solution. Then, the plate was read in an ELISA reader (Bio-Tek Instruments) at $570 \mathrm{~nm}$.

\section{DNA content}

After 14 days of culture, cells were washed with PBS, homogenized in $1 \mathrm{~mL}$ of MilliQ water, frozen, and thawed twice before analysis. The analysis was performed by using a PicoGreen dsDNA quantification kit (Molecular Probes) following the manufacturer's instructions. Briefly, $100 \mu \mathrm{L}$ of the DNA sample or standard was incubated with $100 \mu \mathrm{L}$ of a working solution in the dark for $10 \mathrm{~min}$. Then, the DNA content was measured using a fluorescence microplate reader (Bio-Tek Instruments) with an excitation filter $485 \mathrm{~nm}$ and emission filter $530 \mathrm{~nm}$.

\section{ALP activity}

ALP activity assay was performed after 14 days of culture according to a previous study on MC3T3-E1 cells. ${ }^{16}$ Cells were harvested the same way as the samples for the DNA assay. For the analysis, $80 \mu \mathrm{L}$ of a sample or standard (serial dilutions of 4-nitrophenol at the concentrations of $0-25 \mathrm{nM}$ ) and $20 \mu \mathrm{L}$ of a buffer solution (0.5 M 2-amino-2-methyl-1propanol) were added into a 96-well plate. Then, $100 \mu \mathrm{L}$ of a substrate solution (p-nitrophenyl phosphate) was added to all the wells and subsequently, the mixture was incubated at $37^{\circ} \mathrm{C}$ for $1 \mathrm{~h}$. After incubation, the reaction was stopped by adding $100 \mu \mathrm{L}$ of $0.3 \mathrm{M} \mathrm{NaOH}$, and the ALP activity was measured in an ELISA reader (Bio-Tek Instruments) at $405 \mathrm{~nm}$ and it was normalized to the DNA content.

\section{RNA isolation}

After 14 days of culture, the total RNA was isolated from the cells in 12-well plates $(n=3)$ using the TRIzol ${ }^{\circledR}$ reagent 
(Invitrogen) according to the manufacturer's instructions. In brief, after removing the culture medium, $1 \mathrm{~mL}$ of the TRIzol ${ }^{\circledR}$ reagent was added to each well for cell homogenization. The cell extract was mixed vigorously with $0.2 \mathrm{~mL}$ of chloroform and centrifuged at $12,000 \mathrm{~g}$ for $15 \mathrm{~min}$ at $4^{\circ} \mathrm{C}$. The aqueous phase of the sample was collected and mixed with $0.5 \mathrm{~mL}$ of $100 \%$ isopropanol. After incubation at room temperature for $10 \mathrm{~min}$, the extract was centrifuged and then washed with $75 \%$ ethanol. Successively, the RNA pellet was dissolved in RNase-free water and the concentration and purity were determined by NanoDrop (ND-2000; Thermo Scientific). RNA samples with an A260/A280 ratio <1.8 were excluded.

\section{Reverse transcriptase reaction}

After the isolation of RNA, the reverse transcriptase reaction was performed with Superscript ${ }^{\mathrm{TM}}$ III First-strand Synthesis System for RT-PCR (Invitrogen) following the manufacturers' protocol. Briefly, the collected $1 \mu \mathrm{g}$ total RNA in $8 \mu \mathrm{L}$ RNA free water, $0.5 \mu \mathrm{L}$ random hexamers, $0.5 \mu \mathrm{L}$ oligo (dT) primers, and $1 \mu \mathrm{L}$ of dNTPs were incubated for $5 \mathrm{~min}$ at $65^{\circ} \mathrm{C}$. Then, the following components were subsequently added: $2 \mu \mathrm{L}$ reaction buffer, $4 \mu \mathrm{L} 25 \mathrm{~mm} \mathrm{MgCl}_{2}, 2 \mu \mathrm{L}$ $0.1 \mathrm{~m} \mathrm{DTT}, 1 \mu \mathrm{L}$ RNase OUT, and $1 \mu \mathrm{L}$ Superscript III RT. The reaction mix was incubated for $10 \mathrm{~min}$ at $25^{\circ} \mathrm{C}$ for further primer annealing, $50 \mathrm{~min}$ at $50^{\circ} \mathrm{C}$ for reverse transcription, and $5 \mathrm{~min}$ at $85^{\circ} \mathrm{C}$ to terminate the reaction. This cDNA solution was stored at $-20^{\circ} \mathrm{C}$ for further use.

\section{Real-time PCR}

The cDNA was further amplified and the expression of specific genes was quantified using the IQ SYBR Green Supermix PCR kit (BioRad) in a real-time PCR (BIORAD, CFX96 ${ }^{\mathrm{TM}}$ real-time system). For this reaction, $2 \mu \mathrm{L}$ cDNA, $1.5 \mu \mathrm{L}$ former and $1.5 \mu \mathrm{L}$ reverse primers, $12.5 \mu \mathrm{L}$ master mix, and $7.5 \mu \mathrm{L}$ RNase-free water were mixed and centrifuged. Subsequently, PCR was performed at $60^{\circ} \mathrm{C}$. Osteoblastic differentiation-related primers were used to check the gene expression at the RNA level, including bone sialoprotein $(B S P), O C$, and osteopontin $(O P N)$. GAPDH was referred as the housekeeping gene (sequences in Table 1). Specific sense and antisense primers for the genes were designed according to published cDNA sequences of GenBank. The specificity of the primers was tested separately before the reaction and the negative control consisted of RNase-free water. The calculation of gene expression was performed via the $2^{-\Delta \Delta C t}$ method. ${ }^{17}$

\section{Statistical analysis}

The calcium content and ALP activity assays were always performed in quadruplicate and samples for real-time PCR were assayed in triplicate. All data were reported as mean \pm SD. Statistical analysis of quantitative data was performed by using one-way ANOVA combined with the Student-Newman-Keuls multiple comparison post test and the significance level was 0.05 (Instat 3.05; GraphPad Software, Inc.).

\section{Results \\ Cell morphology}

To evaluate the effect of the passage number and culturing supplements on the morphology of MC3T3-E1 cells, morphological observation was performed on days 1, 4, and 7. No change in MC3T3-E1 cell morphology was observed among all the passages. On day 1, MC3T3-E1 cells displayed a typical fibroblast-like appearance regardless of culturing supplements (Fig. 1A). On day 4, all the cells exhibited short spindle-shaped morphology and no difference was observed in different groups (Fig. 1B). On day 7, all the cells grew in multilayers and lost the fibroblastic characteristics (Fig. 1C, D). However, most cells in the control and AsAP groups showed a cuboidal appearance (Fig. 1C), while cells in the Dex and AsAP/Dex groups developed an elongated shape (Fig. 1D).

\section{Cell viability}

To detect whether the passage number and culturing supplements influenced the viability of MC3T3-E1 cells, cell survival was assessed after 2 days of culture using a live/ dead assay from passage 22 through passage 50 every 4 passages. Qualitative data (Supplementary Fig. S1; Supplementary Data are available online at www.liebertpub.com/ tec) showed that most cells were identified as live cells (green color) and no difference was observed between the groups.

\section{Calcium content}

To investigate the effect of continuous passaging and culturing supplements on the mineralization of MC3T3-E1 cells, cells were cultured in a control medium, or a control medium containing either AsAP, Dex, or AsAP/Dex for up to 28 days. From passage 22 through passage 50, mineralization was evaluated through quantitative calcium content analysis every four passages on day 14, 21, and 28. Over the first 3 weeks of osteogenic induction, no calcium deposition was detected for any of the groups. By the 28th day of culture, a substantial amount of calcium deposition was detected with cells cultured in AsAP and AsAP/Dex from passage 22 through 30 (Fig. 2). A dramatic decrease in calcium deposition was observed at an increasing passage number, and no calcium deposition at all was found from passage 34 (Fig. 2). The addition of only Dex led to no change

Table 1. Primer Sequences Used for Real-Time Quantitative Polymerase Chain Reaction

\begin{tabular}{lll}
\hline & \multicolumn{1}{c}{ Forward $\left(5^{\prime} \rightarrow 3^{\prime}\right)$} & \multicolumn{1}{c}{ Reverse $\left(5^{\prime} \rightarrow 3^{\prime}\right)$} \\
\hline BSP & TTTATCCTCCTCTGAAACGGT & GTTTGAAGTCTCCTCTTCCTCC \\
OC & CCGGGAGCAGTGTGAGCTTA & TAGATGCGTTTGTAGGCGGTC \\
OPN & GATGAACAGTATCCTGATGCC & TTGGAATGCTCAAGTCTGTG \\
GAPDH & AACGACCCCTTCATTGAC & TCCCACGACATACTCAGCAC \\
\hline
\end{tabular}



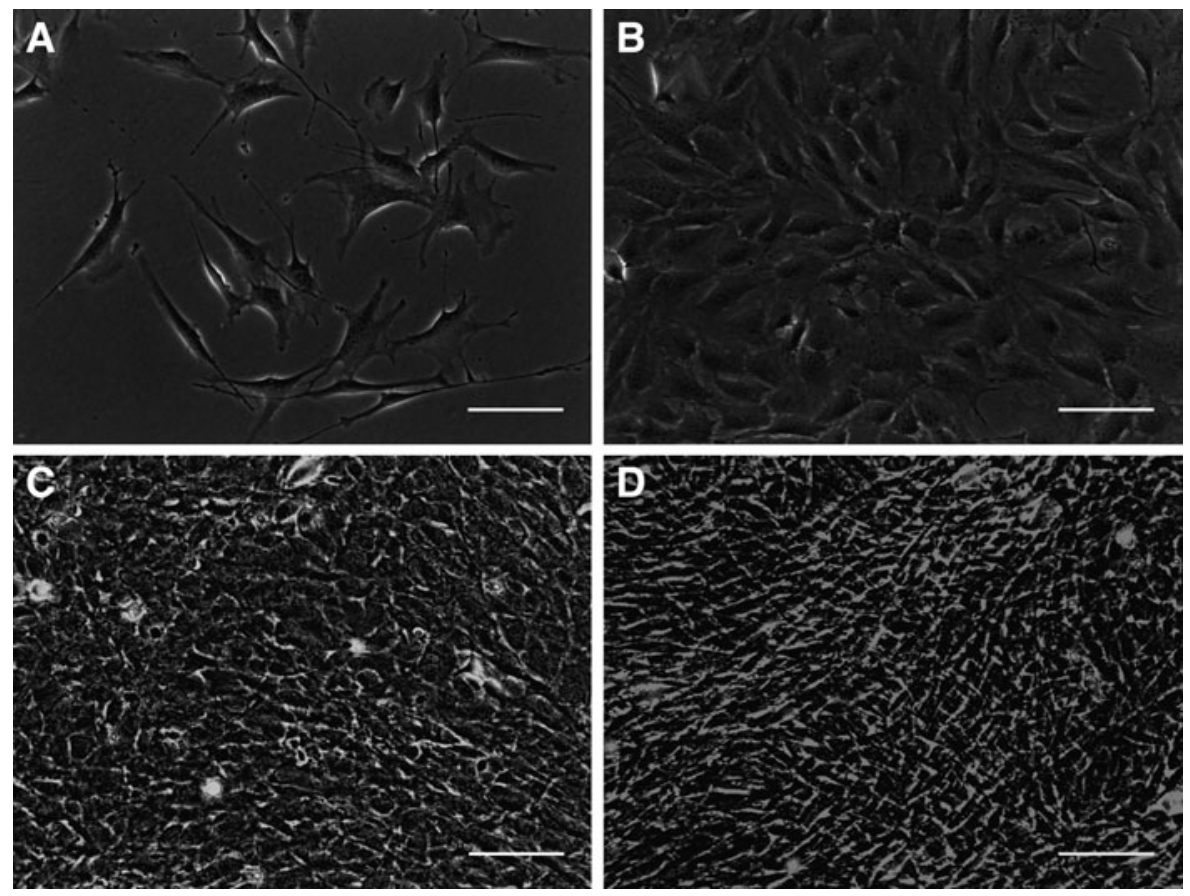

FIG. 1. The effect of culturing supplements on the morphology of MC3T3-E1 cells. On day 1, MC3T3E1 cells exhibited a typical fibroblast-like appearance regardless of culturing supplements (A). On day 4 , all the cells displayed short spindle-shaped morphology and no difference was observed in different groups (B). On day 7, all the cells grew in multilayers and lost the fibroblastic characteristic (C, D).

Most cells in control and ascorbic acid (AsAP) groups showed a cuboidal appearance $(\mathrm{C})$, while cells in dexamethasone (Dex) and AsAP/Dex groups developed an elongated shape (D). Scale bars: $100 \mu \mathrm{m}$. in the calcium content as there was no significant difference with the control culture (Fig. 2). In contrast, the mineralization capability of MC3T3-E1 cells was enhanced significantly by the addition of AsAP or AsAP/Dex as compared to the control and Dex groups $(p<0.05)$. Moreover, the enhanced calcium content was more prominent in cell cultures supplemented with AsAP/Dex than only AsAP (Fig. 2). At passage 22 and 26, the addition of AsAP/Dex stimulated significantly more calcium deposition than that in the group with only AsAP $(p<0.01)$, with an increase of approximately $40 \mu \mathrm{g} / \mathrm{mL}$ for each passage (Fig. 2).

\section{ALP activity}

To evaluate the effect of continuous passaging and culturing supplements on the osteogenic capacity of MC3T3-E1

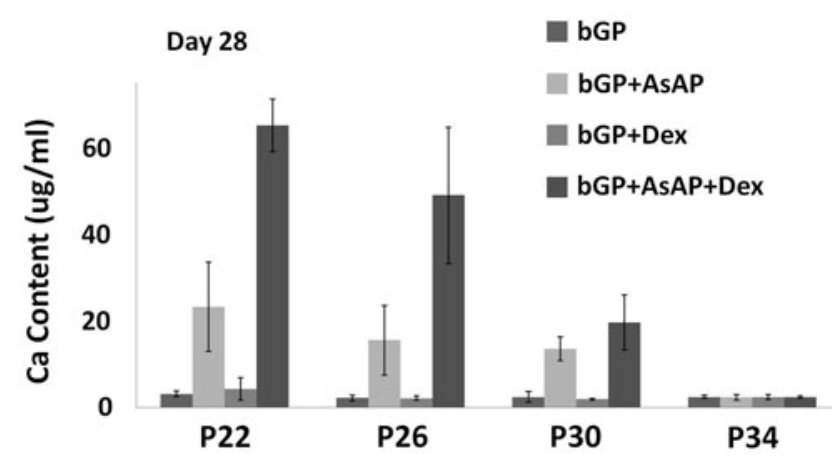

FIG. 2. The effect of passage number and culturing supplements on the mineralization capacity of MC3T3-E1 cells. The results indicate that MC3T3-E1 cells display a significant decrease in the calcium content during the process of continuous passage and lose the osteogenic capacity within 34 passages. The combination of AsAP and Dex stimulates a higher calcium deposition by the cells than a single factor does. Error bars represent standard error of the mean $(n=4)$. cells, the ALP activity on day 14 was further measured at passage 22, 26, and 30. Comparable to the calcium content study, Figure 3 showed significantly higher levels of the ALP activity for cells at passage 22 and 26 versus cells at passage 30 . In contrast to the calcium content study, the addition of AsAP or AsAP/Dex resulted in a lower ALP activity in MC3T3-E1 cells compared to the control group and Dex alone group (Fig. 3).

\section{Gene expression}

To verify the effect of osteogenic culture supplements on the osteogenic capacity of MC3T3-E1 cells, expression of

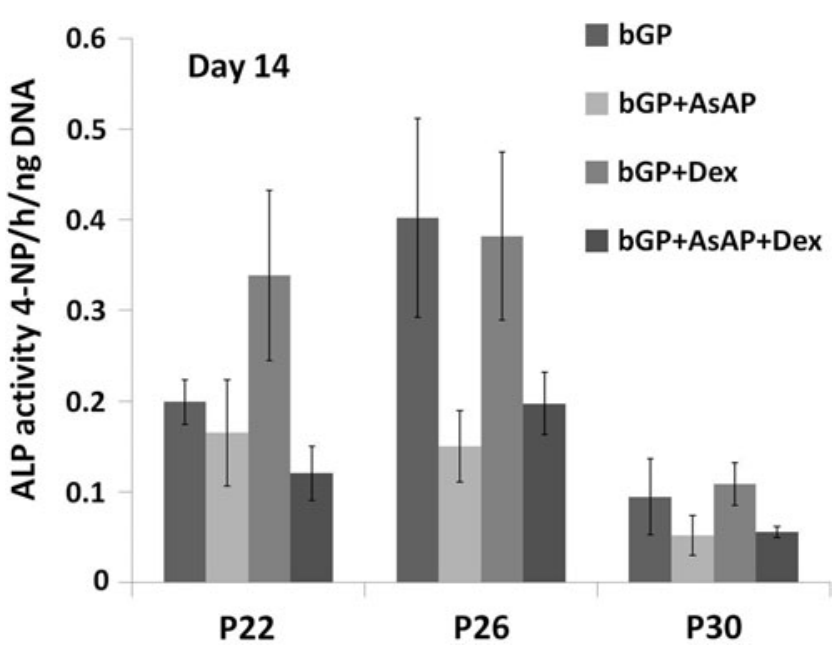

FIG. 3. The effect of passaging and culturing supplements on the alkaline phosphatase (ALP) activity of MC3T3-E1 cells. Significantly higher levels of the ALP activity were observed for cells at passage 22 and 26 versus cells at passage 30. The addition of AsAP or AsAP/Dex did not stimulate a higher ALP activity in MC3T3-E1 cells. 
genes involved with osteogenesis (BSP,OC, and OPN) was further determined by performing real-time quantitative RTPCR on the cells cultured for 14 days. Quantification of gene expression was only performed at passage 22, 26, and 30 since these cells still possess the capacity to mineralize. The Dex alone group was excluded because no calcium deposition was detected for this group. The highest expression levels of BSP were seen in the AsAP/Dex groups, which displayed a significantly higher expression of BSP expression than the AsAP alone groups (Fig. 4A). For OC gene expression, a significant increase was seen when AsAP or AsAP/ Dex was added compared to the control group (Fig. 4B). There was also an increase in the AsAP/Dex group compared with the AsAP alone group, but this increase was not significant. As shown in Figure $4 \mathrm{C}$, a significantly higher expression of OPN was found by the addition of AsAP/Dex compared to the control and AsAP groups at passage 22 .

\section{Discussion}

The purpose of the current study was to investigate the effects of Dex and AsAP on the mineralization of MC3T3-E1 cells at different passages. We hypothesized that the osteogenic ability was compromised in MC3T3-E1 cells undergoing continuous passaging. Secondly, it was supposed that a combination of osteogenic supplements would further stimulate mineralization. Our results demonstrated a significantly decreased mineralization capacity of MC3T3-E1 cells during passaging without negatively affecting their viability (determined by deposited calcium content). At 34 in vitro passages, the mineralization capacity of the cells was observed to be exhausted as there was no calcium deposition. The osteogenic ability of MC3T3-E1 cells, as induced by the combination of AsAP and Dex and measured by the calcium content and expression of osteogenetic genes (BSP and OPN), was significantly higher than the traditional addition of AsAP alone.

The observed reduced osteogenic capacity during passaging is in agreement with previous research. ${ }^{9}$ Chung et al. reported that the osteoblastic function of MC3T3-E1 cells from late passage $(>65)$ was significantly inhibited as compared to the early passage $(<20) .{ }^{9}$ Comparable to their results, significantly higher levels of the ALP activity for cells at passage 22 and 26 were detected in comparison to cells at passage 30. Our data also indicate that MC3T3-E1 cells are losing their functional capacity of mineralized nodule formation between passage 30 to 34 without affecting their morphology and viability. The effect of passage number on cell lines is not limited to MC3T3-E1 cells. It has been reported that the passaging process significantly influences the physiological development and transport properties of the Caco-2 cell line. ${ }^{18,19}$ This change in behavior can be caused by genetic mutations, ${ }^{8,18}$ or due to a positive selection of fastgrowing cells from the population of heterogeneous cell lines during passaging, ${ }^{8,18}$ since heterogeneity has been observed in the MC3T3-E1 cell line as well as in other cell lines. ${ }^{4,20}$

Recently, more and more national and international collaborations take place, which favor scientific discovery significantly. The exchange of cell lines between laboratories is frequently part of the collaborative efforts. However, this harbors the risk that important information, like the passage number of the cells, gets lost. Our results suggest that over-
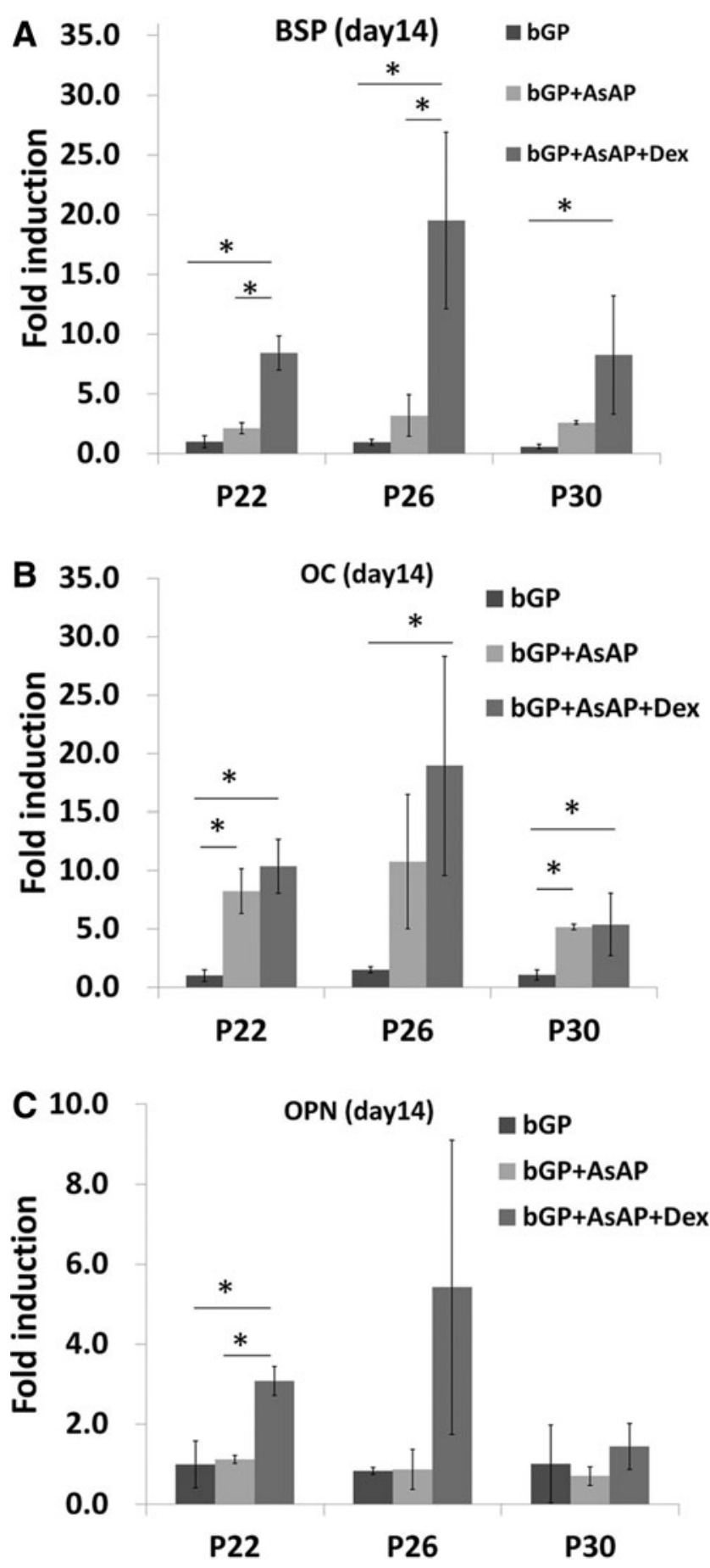

FIG. 4. The effect of culturing supplements on the expression of bone sialoprotein $(B S P)$, osteocalcin $(O C)$, and osteopontin $(O P N)$. Cells cultured with AsAP/Dex displayed significantly higher levels of $B S P$, compared to those of the AsAP group (A). A significant increase in OC gene expression was observed when AsAP or AsAP/Dex was added compared to the control group (B). The addition of AsAP/ Dex significantly increased the expression of OPN compared to the control group at passage $22(\mathrm{C}) .{ }^{*} p<0.01$; error bars represent standard error of the mean $(n=3)$. 
passaged cells and cell lines without a defined passage number should not be used.

The other purpose of the current study was to further optimize osteogenic culture conditions of MC3T3-E1 cells, of which, the traditionally supplemented osteogenic inducer is AsAP. $^{4}$ In contrast, rat bone marrow cells mineralize only when Dex is added to the culture medium. ${ }^{6}$ Therefore, we decided to assess whether Dex can further optimize the osteogenic induction of MC3T3-E1 cells. The used doses were chosen according to previous studies, in which, $50 \mu \mathrm{g} / \mathrm{mL}$ AsAP triggered an optimal increase in osteogenic differentiation of osteoblast-like cells from the human facial bone ${ }^{21}$ and $10 \mathrm{nM}$ Dex was the optimal condition for mineralization of human mesenchymal stem cells. ${ }^{22}$ However, it has to be pointed out that different species may have their optimal doses for osteogenic culture supplements. Therefore, different doses of AsAP and Dex for mouse MC3T3-E1 cells may give rise to different results. For RT-PCR analysis, BSP and $O C$ were selected as discriminatory genes, because a good correlation has been observed between their mRNA expressions and the ability of a given subclone to form a mineralized extracellular matrix in culture. ${ }^{4}$

Our results indicated that the combination of AsAP and Dex promotes osteogenic ability of MC3T3-E1 cells when compared to AsAP alone, as shown by the significantly higher calcium deposition. These results highlight the importance of Dex in influencing the mineralization of MC3T3E1 cells. However, no change in mineralization was observed in the Dex alone group as compared to the control, indicating Dex has an indirect effect on mineralization of MC3T3-E1 cells. This investigation was described similar by others. ${ }^{14}$ Roberts et al. reported that Dex augmented osteogenic differentiation of periosteal-derived cells in combination with all trans-retinoic acid (atRA) and bone morphogenic protein 2 (BMP2), but had no direct effect on the differentiation. ${ }^{14}$ This phenomenon can be interpreted that Dex acts directly and/or indirectly to promote the osteoprogenitor cell numbers in cultures. ${ }^{23}$ The higher cell numbers stimulated by Dex can also explain the elongated cell shape in Dex groups on day 7 , as there is a significant amount of overlap between the cells.

Another surprising phenomenon that has to be pointed out is, in contrast to the mineralization result, that the addition of AsAP or AsAP/Dex stimulated a lower ALP activity in MC3T3-E1 cells compared to the control group and Dex alone group. This is comparable to another recently published study, in which, inositol hexakisphosphate (IP6) decreased mineralization and gene expression of osteoblast markers in MC3T3-E1 cells without negatively affecting the ALP activity. ${ }^{16}$ Besides, Beck et al. reported that ALP levels can be dissociated from mineralization of MC3T3-E1 cells, as mineralization can occur in the absence of ALP induction. ${ }^{19}$ Cell lines exhibiting severe repression of the ALP activity can differentiate to a mineral-secreting phenotype. ${ }^{19}$ These results may indicate that ALP activity alone is not sufficient for the determination of the osteogenic capacity of MC3T3E1 cells.

In conclusion, our findings indicate that (1) the passage number of MC3T3-E1 cells should be clearly defined in all scientific studies; (2) the use of MC3T3-E1 cells for osteogenic differentiation should be avoided after passage 30; (3) AsAP and Dex are suggested as the supplements for the osteogenic induction of MC3T3-E1 cells.

\section{Acknowledgment}

Xiangzhen Yan and Wanxun Yang are grateful to the program of China Scholarship Council for the financial support (No. 2010622061, 2010627030). This work was supported by the Royal Netherlands Academy of Arts and Sciences (KNAW; project number PSA 08-PSA-M-02).

\section{Disclosure Statement}

No competing financial interests exist.

\section{References}

1. Sudo, H., Kodama, H.A., Amagai, Y., Yamamoto, S., and Kasai, S. In vitro differentiation and calcification in a new clonal osteogenic cell line derived from newborn mouse calvaria. J Cell Biol 96, 191, 1983.

2. Owen, T.A., Aronow, M., Shalhoub, V., Barone, L.M., Wilming, L., Tassinari, M.S., et al. Progressive development of the rat osteoblast phenotype in vitro: reciprocal relationships in expression of genes associated with osteoblast proliferation and differentiation during formation of the bone extracellular matrix. J Cell Physiol 143, 420, 1990.

3. Quarles, L.D., Yohay, D.A., Lever, L.W., Caton, R., and Wenstrup, R.J. Distinct proliferative and differentiated stages of murine MC3T3-E1 cells in culture: an in vitro model of osteoblast development. J Bone Miner Res 7, 683, 1992.

4. Wang, D., Christensen, K., Chawla, K., Xiao, G., Krebsbach, P.H., and Franceschi, R.T. Isolation and characterization of MC3T3-E1 preosteoblast subclones with distinct in vitro and in vivo differentiation/mineralization potential. J Bone Miner Res 14, 893, 1999.

5. Kretlow, J.D., Jin, Y.Q., Liu, W., Zhang, W.J., Hong, T.H., Zhou, G., et al. Donor age and cell passage affects differentiation potential of murine bone marrow-derived stem cells. BMC Cell Biol 9, 60, 2008.

6. Ter Brugge, P.J., and Jansen, J.A. In vitro osteogenic differentiation of rat bone marrow cells subcultured with and without dexamethasone. Tissue Eng 8, 321, 2002.

7. Wan Safwani, W.K., Makpol, S., Sathapan, S., and Chua, K.H. The changes of stemness biomarkers expression in human adipose-derived stem cells during long-term manipulation. Biotechnol Appl Biochem 58, 261, 2011.

8. Hughes, P., Marshall, D., Reid, Y., Parkes, H., and Gelber, C. The costs of using unauthenticated, over-passaged cell lines: how much more data do we need? Biotechniques 43, 575, 2007.

9. Chung, C.Y., Iida-Klein, A., Wyatt, L.E., Rudkin, G.H., Ishida, K., Yamaguchi, D.T., et al. Serial passage of MC3T3-E1 cells alters osteoblastic function and responsiveness to transforming growth factor-beta1 and bone morphogenetic protein-2. Biochem Biophys Res Commun 265, 246, 1999.

10. Peterson, W.J., Tachiki, K.H., and Yamaguchi, D.T. Serial passage of MC3T3-E1 cells down-regulates proliferation during osteogenesis in vitro. Cell Prolif 37, 325, 2004.

11. Song, I.H., Caplan, A.I., and Dennis, J.E. In vitro dexamethasone pretreatment enhances bone formation of human mesenchymal stem cells in vivo. J Orthop Res 27, 916, 2009.

12. Cheng, S.L., Yang, J.W., Rifas, L., Zhang, S.F., and Avioli, L.V. Differentiation of human bone marrow osteogenic stromal cells in vitro: induction of the osteoblast phenotype by dexamethasone. Endocrinology 134, 277, 1994.

13. Mostafa, N.Z., Uludag, H., Varkey, M., Dederich, D.N., Doschak, M.R., and El-Bialy, T.H. In vitro osteogenic induction of human gingival fibroblasts for bone regeneration. Open Dent J 5, 139, 2011. 
14. Roberts, S.J., Chen, Y., Moesen, M., Schrooten, J., and Luyten, F.P. Enhancement of osteogenic gene expression for the differentiation of human periosteal derived cells. Stem Cell Res 7, 137, 2011.

15. Jin, Z.L., Zhang, Y.K., Sun, H.Y., Lin, Z., Bi, Y.C., Duan, Y.Z., et al. Osteogenic-related gene expression profiles of human dental follicle cells induced by dexamethasone. Acta Pharmacol Sin 29, 1013, 2008.

16. Arriero, M.D., Ramis, J.M., Perello, J., and Monjo, M. Differential response of MC3T3-E1 and human mesenchymal stem cells to inositol hexakisphosphate. Cell Physiol Biochem 30, 974, 2012.

17. Livak, K.J., and Schmittgen, T.D. Analysis of relative gene expression data using real-time quantitative PCR and the 2(-Delta Delta C(T)) Method. Methods 25, 402, 2001.

18. Kono, R., Okuno, Y., Inada, K., Tokuda, A., Hashizume, H., Yoshida, M., et al. A Prunus mume extract stimulated the proliferation and differentiation of osteoblastic MC3T3-E1 cells. Biosci Biotechnol Biochem 75, 1907, 2011.

19. Beck, G.R., Jr., Sullivan, E.C., Moran, E., and Zerler, B. Relationship between alkaline phosphatase levels, osteopontin expression, and mineralization in differentiating MC3T3-E1 osteoblasts. J Cell Biochem 68, 269, 1998.

20. van Steenbrugge, G.J., van Uffelen, C.J., Bolt, J., and Schroder, F.H. The human prostatic cancer cell line LNCaP and its derived sublines: an in vitro model for the study of androgen sensitivity. J Steroid Biochem Mol Biol 40, 207, 1991.
21. Pradel, W., Mai, R., Gedrange, T., and Lauer, G. Cell passage and composition of culture medium effects proliferation and differentiation of human osteoblast-like cells from facial bone. J Physiol Pharmacol 59 Suppl 5, 47, 2008.

22. Mostafa, N.Z., Fitzsimmons, R., Major, P.W., Adesida, A., Jomha, N., Jiang, H., et al. Osteogenic differentiation of human mesenchymal stem cells cultured with dexamethasone, vitamin $\mathrm{d} 3$, basic fibroblast growth factor, and bone morphogenetic protein-2. Connect Tissue Res 53, 117, 2012.

23. Aubin, J.E. Osteoprogenitor cell frequency in rat bone marrow stromal populations: role for heterotypic cell-cell interactions in osteoblast differentiation. J Cell Biochem 72, 396, 1999.

Address correspondence to: John A. Jansen, PhD, DDS Department of Biomaterials Radboud University Nijmegen Medical Centre PO Box 9101 Nijmegen $6500 \mathrm{HB}$ The Netherlands

E-mail: j.jansen@dent.umcn.nl

Received: July 5, 2012

Accepted: June 6, 2013

Online Publication Date: July 29, 2013 